\title{
Heterogeneidade espaço-temporal ambiental de igarapés em um ecótono Cerrado-Caatinga
}

\author{
Daniel Silas Verasa, Lázaro Carlos Medeiros-Françab* ${ }^{b^{*}}$ Carlos Augusto Silva de Azêvedo ${ }^{a}$ \\ a Programa de Pós-Graduação em Biodiversidade, Ambiente e Saúde, Universidade Estadual do Maranhão, Caxias, 65600-000, \\ Maranhão, Brasil. \\ ${ }^{b}$ Laboratório de Entomologia Aquática, Centro de Estudos Superiores de Caxias, 65.600-000, Maranhão, Brasil. \\ *lazarobiocx@gmail.com
}

Recebido: 19 janeiro 2018 / Aceito: 13 agosto 2018 / Publicado online: 12 setembro 2018

\begin{abstract}
Resumo
A heterogeneidade espaço-temporal do ambiente é um fator importante para a estruturação da biodiversidade dos ecossistemas, incluindo os sistemas lóticos que têm sofrido fortes influência do uso e ocupação do solo. Nesse sentido, o presente trabalho teve por objetivo identificar se houve diferença espaço-temporal das variáveis estruturais e físico-químicas entre os riachos da Área de Proteção Ambiental do Inhamum, bem como se ocorreu diferença de heterogeneidade ambiental entre as amostras durante o período de 2012 a 2015. A ordenação indicou efeitos espaço temporais sobre os riachos, pois houve a formação de dois grupos, o primeiro formado pelas amostras de 2012-2013 e a outra de 2014-2015, mas que não apresentaram diferenças quanto sua heterogeneidade. A temperatura, condutividade, profundidade, largura e vazão foram importantes na estruturação dos riachos, mas em decorrência de estarem em uma área com uma ampla cobertura vegetal e alta integridade de habitat, houve uma pequena amplitude de variação nos valores dessas variáveis. Tais resultados demonstram a importância da manutenção da cobertura vegetal para garantir a integridade de habitat e prover a conservação das características ambientais de riachos em biomas tropicais.
\end{abstract}

Palavras-chave: conservação, integridade de habitat, monitoramento, sistemas lóticos.

\section{Environmental spatial-temporal heterogeneity of streams in a Cerrado-Caatinga ecotone}

\begin{abstract}
Environmental spatial-temporal heterogeneity is an important factor for structuring the biodiversity of ecosystems, including lotic systems that have been strongly influenced by land use and occupation. Thus, the present study aimed to identify if there was a spatial-temporal difference of the structural and physical-chemical variables which the streams of the Environmental Protection Area of the Inhamum, as well as if there was difference of environmental heterogeneity which the samples during the period of 2012 to 2015 . The ordernation indicated temporal effects on the streams, because there were two groups formed, the first formed by the samples of 2012-2013 and the other by 2014-2015, but which did not present differences as to their heterogeneity. Temperature, conductivity, depth, width and flow were important in the structuring of the streams, but due to being in an area with a wide canopy cover and high habitat integrity, there was a small range of variation in the values of these variables. These results demonstrate the importance of the maintenance of the canopy cover to guarantee the habitat integrity and to provide the conservation of the environmental characteristics of streams in tropical biomes.
\end{abstract}

Keywords: conservation, habitat integrity, monitory, lotic systems.

\section{Introdução}

A estrutura e a função de ecossistemas lóticos apresentam um padrão de heterogeneidade espacial do habitat, assim como uma variabilidade temporal das condições ambientais (Poff e Ward, 1990; Vannote, et al. 1980). Essa variação geralmente se apresenta em forma de gradiente de suas condições, recursos e estrutura que tendem a se modificar ao longo do tempo por mecanismo de sucessão ecológica (Fisher, et al. 2011; Southwood, 1977), bem como por pressão das atividades antrópicas (Chauran e Verma, 2015; Conroy et al. 2016). Essas mudanças temporais e espaciais do habitat são importantes drivers para determinar a estrutura e composição de espécies das comunidades biológicas que estão presentes nesses ecossistemas (Castro et al. 2018; Silva et al. 2018).

Contemporaneamente, as atividades antropogênicas têm sido a principal causa de mudança nos padrões de heterogeneidade espacial e variabilidade temporal do habitat 
dos diferentes ecossistemas (Chauran e Verma, 2015; Frascareli et al. 2015). No caso dos ecossistemas lóticos a modificação nesses padrões ocorre principalmente em decorrência da perda da mata ripária (Siegloch et al. 2016), que funciona como uma zona de amortecimento que reduz a entrada de sedimentos, lixiviação de agrotóxicos e fertilizantes, assim como mantém a temperatura constante da água e a entrada de matéria orgânica alóctone para o leito dos riachos (Siegloch et al. 2016; Oliveira et al. 2017).

Considerando a importância do padrão temporal e espacial da qualidade da água, tanto para a sobrevivência da biota aquática, quanto para seus diferentes usos para a sociedade, tem sido realizado estudos caracterizando as condições de riachos, rios e represas de diferentes bacias hidrográficas (Fisher et al. 2011; Frascareli et al. 2015). Esses estudos são realizados em locais com forte pressão do uso e ocupação do solo, fato que leva a modificações mais conspícuas da estrutura ambiental devido à retirada da cobertura vegetal das margens dos corpos hídricos (OlivaGonzáles et al. 2011; Finkler et al. 2016; Rocha e Pereira, 2016).

Portanto, se faz necessário o acompanhamento das áreas de proteção ambiental (APA), pois essas são destinadas a conservação de nascentes de rios, as quais podem também sofrer pressão das ações antrópicas que põem em risco os recursos hídricos da bacia (Leitão et al., 2015; Marmotel e Rodrigues, 2015; Pieroni et al. 2015). E uma vez que à medida que se altera a estrutura do ambiente, também ocorrem modificações nas características físico-químicas, como temperatura, oxigênio dissolvido, $\mathrm{pH}$ e biológicas da água (Monteiro-Júnior et al. 2013; Monteiro Júnior et al. 2014). Por isso muitas pesquisas têm utilizado protocolos para determinar a integridade ambiental do ecossistema
(Nessimian et al. 2008).

Dessa forma é importante o monitoramento da qualidade da água, atividade que apresenta difícil análise e interpretação dos dados em decorrência da quantidade de variáveis, principalmente quando o levantamento ocorre por muitos anos (Finkler et al. 2016; Rocha e Pereira, 2016). Nesse sentido é que diversos trabalhos têm utilizado as análises multivariadas que possibilitam a redução no número de variáveis sem a perda significativa de informação (Guedes et al. 2012; Santi et al. 2012; Finkler et al. 2016; Rocha e Pereira, 2016;).

Desta forma o presente trabalho buscou identificar se houve diferença espaço-temporal das variáveis estruturais e físicoquímicas entre os riachos da APA do Inhamum, bem como se ocorreu diferença de heterogeneidade ambiental entre as amostras durante o período de 2012 a 2015.

\section{Material e Métodos}

\section{Área de estudo}

A Área de Proteção Ambiental Municipal do Inhamum é considerada Patrimônio Municipal de Caxias, está localizada entre as coordenadas $04^{\circ} 53^{\prime} 30^{\prime \prime}$ de Latitude S e 43 $24^{\prime}$ '53" de Longitude W (Figura 1), próximo a zona urbana da qual é separada pela BR 316 e tem aproximadamente 3 mil hectares. A fitofisionomia predominante é a de Cerrado, com dois estratos, um arbóreo/arbustivo e outro graminoso em áreas planas, há diversas nascentes que formam lagoas, brejos e córregos (Neres e Conceição, 2010; Medeiros, 2015). O município de Caxias apresentava para o ano de 2010 uma população de 155.129 pessoas, e densidade populacional de $30,12 \mathrm{hab} / \mathrm{Km}^{2}$ (IBGE, 2018).

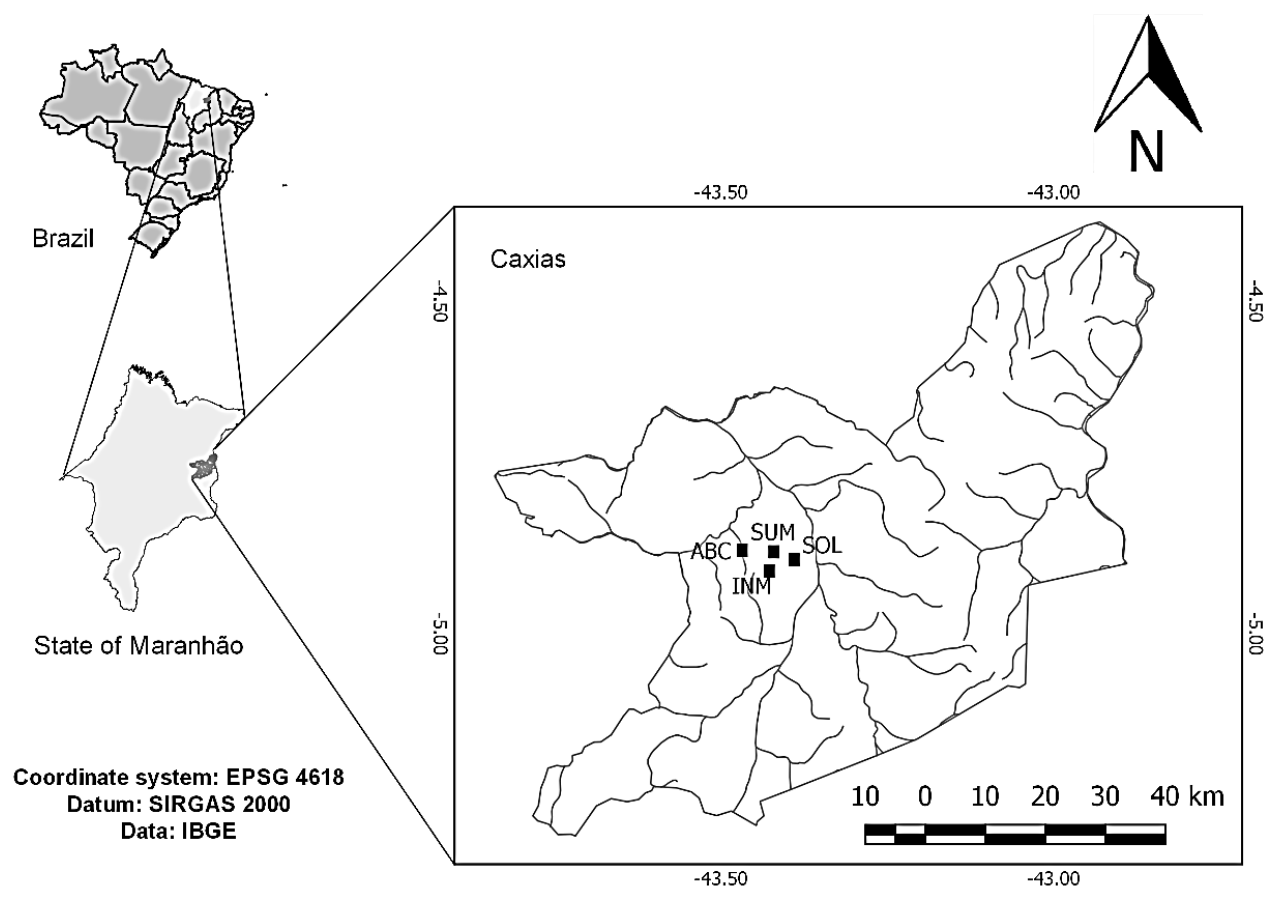

Figura 1. Riachos amostrados na Área de Proteção Ambiental do Inhamum, Caxias, Maranhão, Brasil. Pontos amostrados: Areia Branca (ABC), Inhamum (INM), Soledade (SOL), Sumidouro do Padre (SUM). 


\section{Coleta de dados e analises físico-químicos}

Os parâmetros ambientais e físico-químicos medidos foram: largura, profundidade, velocidade, vazão, índice de integridade de habitat, potencial hidrogeniônico $(\mathrm{pH})$, condutividade elétrica (CE), temperatura. As amostragens foram realizadas entre os anos de 2012 a 2015, na estação seca (junho a novembro). A velocidade e a vazão foram mensuradas pelo método de Craig (1987), consistindo em tomar duas medidas da profundidade da água, utilizando uma régua de aço inoxidável. A velocidade foi estimada pela fórmula: $\mathrm{V}=\sqrt{ } 2 \mathrm{gD}$, considerando $\mathrm{V}=$ velocidade da água, $\mathrm{g}=$ força da gravidade $(19,6), \mathrm{D}=$ distância da água sobre a régua. A vazão foi estimada pela fórmula: Vazão $=$ largura $\mathrm{x}$ velocidade da água $\mathrm{x}$ profundidade. $\mathrm{O} \mathrm{pH}$ foi medido pelo pHmetro marca pHtek, a condutividade elétrica pelo Conductivity Meter CD-4301 (Lutron), a temperatura $\left({ }^{\circ} \mathrm{C}\right)$ com um termômetro digital.

Para amostragem das características estruturais dos riachos, no ano de 2016 foi aplicado o Índice de Integridade de Habitat (IIH), esse índice leva em consideração a avaliação de 12 características das condições ambientais, assim realizase uma avaliação visual em relação ao uso da terra, zona ribeirinha, características leito, e morfologia do canal de fluxo, o mesmo tem uma variação de $0-1$, sendo que quanto mais próximo de um mais íntegro é o habitat (Nessimian et al. 2008).

\section{Análise de dados}

As variáveis que apresentaram alta correlação foram retiradas das análises para reduzir problemas com a multicolinearidade. As variáveis limnológicas e estruturais foram sumarizados por período e locais de coleta pela Análise de Componentes Principais (PCA), os dados foram padronizados por apresentarem unidades de grandezas diferentes. A matriz de distância utilizada foi obtida pela distância euclidiana. Para determinar quais componentes principais seriam retidos para análise utilizou-se o modelo de broken-stick.

A análise da ordenação quanto a diferença significative, foi realizada a análise de variância multivariada por permutação (PERMANOVA), e para determinar se havia variabilidade espaço-temporal foi realizado a Análise de dispersão permutacional (PERMDISP) entre as amostras com os dados padronizados. Todas as análises foram realizadas pelas rotinas do programa $\mathrm{R}$ ( $\mathrm{R}$ Development Core Team, 2015), utilizando o pacote vegan para as análises

\section{Resultados e Discussão}

$\mathrm{O}$ índice de integridade de habitat dos riachos variou de 0.81 a 0.92 , a cobertura de dossel teve intervalo entre $82,35 \%$ e $94,12 \%$ (Tabela 1), enquanto os intervalo de profundidade variou de 0,23 a $1,08(0,68 \pm 0.23$, média \pm desvio padrão $)$, largura de 1,34 a 3,41 ( $2,13 \pm 0.59)$, vazão de 0,14 a 1,31 $(0,51 \pm 0,32)$, temperatura de 23,7 a $27,5(25,54 \pm 1.09), \mathrm{pH}$ de 4 a $5,5(4,71 \pm 0.37)$ e CE de 11,1 a $15,6(13,36 \pm 1,64)$.

A variável velocidade apresentou alta correlação $(>0,70)$ com a vazão, por isso não foi utilizada nas análises.
Tabela. 1. Valores de Índice de integridade de Habitat (IIH) e \% Cobertura de Dossel dos igarapés da APA do Inhamum, Caxias, Maranhão, Brasil, 2016.

\begin{tabular}{lcc}
\hline \multicolumn{1}{c}{ Igarapé } & IIH & $\begin{array}{c}\text { \% Cobertura de } \\
\text { dossel }\end{array}$ \\
\hline Inhamum & 0,887 & 94,12 \\
Sumidouro do Padre & 0,915 & 94,12 \\
Soledade & 0,915 & 94,12 \\
Areia Branca & 0,810 & 82,35 \\
\hline
\end{tabular}

A associação dos dois eixos da PCA representou $65,25 \%$ da variação ambiental (Eixo 1: 36,46\% e Eixo 2: 28,80\%), as variáveis que mais contribuíram para formação do primeiro eixo foram profundidade e vazão, relacionadas positivamente e temperatura negativamente com este eixo. A condutividade da água contribuiu positivamente e a temperatura e largura negativamente para a formação do segundo eixo (Figura 2, Tabela 2).

Tabela 2. Variáveis ambientais dos Igarapés da APA do Inhamum, Maranhão, Brasil e suas correlações com os Eixos 1 e 2 da Análise de Componentes Principais (PCA).

\begin{tabular}{lcc}
\hline \multirow{2}{*}{ Variável Ambiental } & \multicolumn{2}{c}{ Loading } \\
\cline { 2 - 3 } Temperatura & Eixo 1 & Eixo 2 \\
pH & $-0,608$ & $-0,649$ \\
Condutividade & $-0,065$ & $-0,316$ \\
Largura & 0,443 & 0,716 \\
Profundidade & 0,243 & $-0,818$ \\
Velocidade & 0,941 & $-0,088$ \\
Vazão & 0,587 & 0,009 \\
Autovalor & 0,829 & $-0,550$ \\
broken-stick & 2,550 & 2,016 \\
\%explicação & 2,592 & 1,592 \\
\hline
\end{tabular}

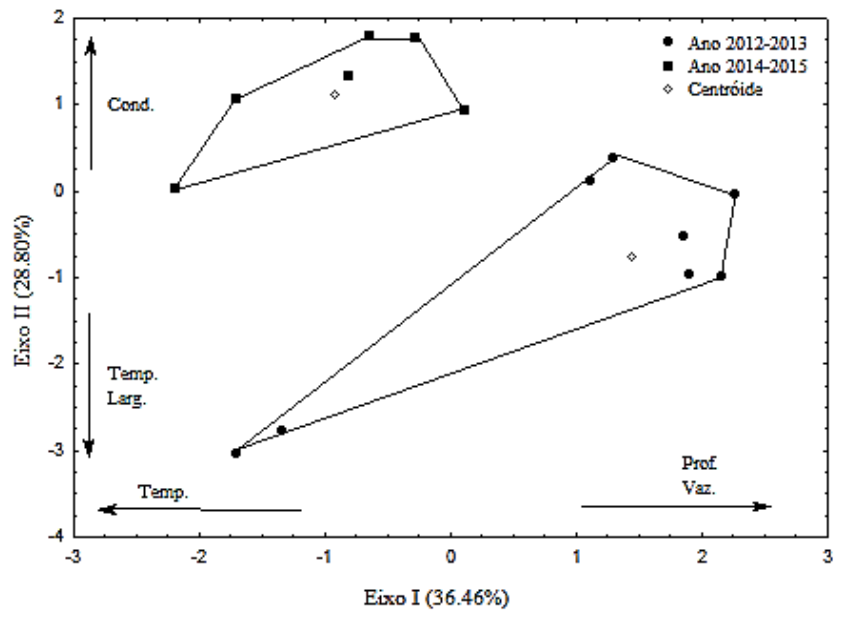

Figura 2. Ordenação das variáveis ambientais por pontos de coleta e ano de coleta dos igarapés da APA do Inhamum, Caxias, Maranhão, Brasil realizada através de Análise de Componentes Principais (PCA). 
O primeiro grupo de igarapés (parte inferior na figura 2) foram constituídos por amostras dos anos de 2012 e 2013, e a segunda ordenação (na parte superior da figura 2) formada pelas amostras de 2014-2015. Assim, houve a separação espaço-temporal significativa entre as variáveis físicoquímicas e estruturais dos riachos amostrados (PERMANOVA, Pseudo-F $(1,14)=5,638, R^{2}=0,287$, $\mathrm{p}=0,002)$.

Nossos resultados indicam que mesmo em riachos íntegros (>0,70) (Carvalho et al. 2013), às variáveis estruturais como profundidade, largura, vazão e as físicoquímicas como temperatura e condutividade podem ser afetados por fenômenos climatológicos (temperatura e pluviosidade). No entanto, essas mudanças apresentam baixo impacto quando comparado com locais que possuem menores valores de integridade, os quais apresentam menor heterogeneidade ambiental (Cunha e Juen, 2017). Assim, verificamos que índices de integridade ambientais podem ser ferramentas efetivas para o monitoramento de Unidades de Conservação.

Não houve diferenças significativa da dispersão das variáveis entre os grupos considerados para período avaliado (PERMDISP, média $=2,428, \mathrm{~F}(1,14)=0,257, \mathrm{p}=0,620)$. Pois a área pesquisada apresenta uma grande proporção de cobertura vegetal, assim observamos uma baixa amplitude de variação tanto das variáveis estruturais quanto físicoquímicas, fato observado em outros estudos que apontam que a maior integridade do habitat conserva a heterogeneidade ambiental (Calvão et al. 2016; Nogueira et al. 2016). Dessa forma corrobora-se a importância da mata ciliar como uma área de amortecimento dos impactos antrópicos ocasionados pelos diferentes usos do solo sobre os ecossistemas naturais, auxiliando na manutenção da biodiversidade (Brasil et al. 2013; Carvalho et al. 2013).

Assim, a heterogeneidade ambiental propiciada pela integridade do habitat pode manter seus diferentes microhabitats que são essenciais para manutenção da riqueza de espécies de forma a conservar a biodiversidade aquática (Nessimian et al. 2008; Dias-Silva et al. 2010). Nesse contexto, o planejamento de uso e ocupação do solo, precisa garantir um manejo que propicie a conservação da cobertura vegetal da APA que é uma condição importante para evitar a homogeneização das variáveis testadas, garantindo os gradientes ambientais do ecossistema onde pode ser atendida uma amplitude de nichos diferentes (Carvalho et al. 2013; Monteiro Júnior et al. 2013).

\section{Conclusões}

Os riachos amostrados apresentaram diferenças espaçotemporais relacionados tanto a suas variáveis estruturais quanto físico-químicas, sendo que a temperatura, condutividade, profundidade, largura e vazão foram as variáveis que mais contribuíram para a variação observada. A Área de Proteção Ambiental do Inhamum, apresenta seu ecossistema íntegro, evidenciando que a integridade de habitat e a cobertura vegetal são variáveis importantes para manutenção das condições ambientais de riachos, podendo ser utilizadas para o monitoramento ambiental.

\section{Referências}

Brasil, L. S., Shimano, Y., Batista, J. D.; Cabette, H. S. R. 2013. Effects of environmental factors on community structure of Leptophlebiidae (Insecta: Ephemeroptera) in Cerrado streams, Brazil. Iheringia, Série Zoologia, 103(3): 260-265.

Calvão L.B., Nogueira D.S., Montag L.F.A., Lopes M.A., Juen L. (2016) Are Odonata communities impacted by conventional or reduced impact logging? Forest Ecology and Management, 382(15): 143-150.

Carvalho, F.G.; Pinto, N.S.; Oliveira-Júnior, J.M.B.; Juen, L. 2013. Efeitos da retirada da vegetação marginal sobre a comunidade de Odonata. Acta Limnologica Brasiliensia, 25(1): 10-18.

Castro, D.M.P.; Dolédec, S.; Callisto. M. 2018. Land cover disturbance homogenizes aquatic insect functional structure in neotropical savanna streams. Ecological Indicators. 84: 573-582.

Chauhan, A.; Verma, S. C. 2015. Impact of Agriculture, Urban and Forest Land Use on Physico-Chemical Properties of Water - A Review. International Journal of Current Microbioly and Applied Sciencies, 4(10): $18-22$.

Conroy, E.; J.N. Turner, J. N.; Rymszewicz, A.; O;Sullivan, J.J.; Bruen, M.; Lawler, D.; Lally, H.; Kelly-Quinn, M. 2016. The impact of cattle access on ecological water quality in streams: Examples from agricultural catchments within Ireland. Science of the Total Environment 547: 17-29.

Cunha E.J.; Juen L. 2017. Impacts of oil palm plantations on changes in environmental heterogeneity and Heteroptera (Gerromorpha and Nepomorpha) diversity. Journal of Insect Conservation 21(1): 111-119.

Dias-Silva K., Cabette H.S.R., Juen L., De Marco P. 2010. The influence of habitat integrity and physical-chemical water variables on the structure of aquatic and semi-aquatic Heteroptera. Zoologia 27(6): 918-930.

Finkler, N.R. Bortolin, T.A.; Cocconi, J.; Mendes, L.A.; Schneider, V.E. 2016. Spatial and temporal assessment of water quality data using multivariate statistical techniques. Ciência e Natura, 38(2): 577-587.

Fisher, J.A.D.; Frank, K.T.; Kostylev, V.E.; Shackell, N.L.; Horsman, T.;Hannah, C.G. 2011. Evaluating a habitat template model's predictions of marine fish diversity on the Scotian Shelf and Bay of Fundy, Northwest Atlantic. ICES Journal of Marine Science, 68(10): 2096-2105.

Frascareli, D.; Beguelli, F.G.S.; Silva, S.C.; Carlos, V.M. 2015. Heterogeneidade espacial e temporal de variáveis limnológicas no reservatório de Itupararanga associadas com o uso do solo na Bacia do Alto Sorocaba-SP. Revista Ambiente e Água, 10(4): 770-781.

Guedes, H.A.S.; Silva, D.D; Elesbon, A.A.A.; Ribeiro, C.B.M.; Matos, A.T. Soares, J.H.P. 2012. Aplicação da análise estatística multivariada no estudo da qualidade da água do Rio Pomba, MG. Revista Brasileira de Engenharia Agrícola e Ambiental, 16(5): 558-563.

IBGE - Instituto Brasileiro de Geografia e Estatística. 2010. Cidades Disponível em: <https://cidades.ibge.gov.br/brasil/ma/caxias/panorama〉. Acesso em 05 jul. 2018.

Leitão, V.S.; Cuba, R.M.F.; Santos, L.P.S.; Neto, A.S.S. 2015. Utilização do índice de qualidade de água (IQA) para monitoramento da qualidade de água em uma área de preservação ambiental. Revista Eletrônica em Gestão, Educação e Tecnologia Ambiental Santa Maria, 19(3): 794-803.

Marmontel, C.V.F.; Rodrigues, V.A. 2015. Parâmetros Indicativos para Qualidade da Água em Nascentes com Diferentes Coberturas de Terra e Conservação da Vegetação Ciliar. Floresta e Ambiente, 22(2): 171-181.

Medeiros R.N. Visões da natureza. In: Sousa, I.G.; Vianna, J.M.; Meneses, R.L. 2015 Cartografias invisíveis: saberes e sentires de Caxias. Academia caxiense de letras, Caxias. 33-34p.

Monteiro-Júnior, C.S. Couceiro, S.R.M.; Hamada, M.; Juen, L. 2013. Effect of vegetation removal for road building on richness and composition of Odonata communities in Amazonia, Brazil. International Journal of Odonatology, 16(2): 135-144.

Monteiro-Júnior, C.S.; Juen, L.; Hamada, N. 2014. Analysis of urban impacts on aquatic habitats in the central Amazon basin: Adult odonates as bioindicators of environmental quality. Ecological Indicators, 48: 303311

Neres, L.P.; Conceição, G.M. 2010. Florística e fitossociologia a Área De Proteção Ambiental Municipal do Inhamum, Caxias, Maranhão, Brasil. Cadernos de Geociências, 7(2): 122-130.

Nessimian, J.L.; Venticinque, E.M.; Zuanon, J.; De Marco Júnior, P.; Gordo, M.; Fidelis, L.; Batista, J.D.; Juen, L. 2008. Land use, habitat integrity, 
and aquatic insect assemblages in Central Amazonian streams. Hydrobiologia, 614: 117-131.

Nogueira D.S.; Calvão L.B.; Montag L.F.A.; Juen L.; De Marco Jr., P. 2016. Little effects of reduced-impact logging on insect communities in eastern Amazonia. Environmental Monitoring and Assessment 188(7): $1-20$.

Oliva-González, S.; Almeida, C.A.; Quintar, S.; Mallea, M.A.; Gonzáles, P.S. 2011. Application of multivariate statistical techniques to evaluate organic pollution on a river in Argentina. Revista Ambiente e Água, 6(3): $27-42$.

Oliveira, D.G.; Vargas, R.R.; Saad, A.R.; Arruda, R.O.M.; Dalmas, F.B.; Azevedo, F.D. 2018. Land use and its impacts on the water quality of the Cachoeirinha Invernada Watershed, Guarulhos (SP). Ambiente \& Água - An Interdisciplinary Journal of Applied Science. 13(1): 1-17.

Pieroni, J.P.; Branco, K.G.R.; Inachvili, I.; Ferreira, G.C. 2015. Monitoramento sazonal da qualidade da água, na sub bacia hidrográfica do córrego água limpa, em seu trecho afetado pela mineração de níquel, no município de Pratápolis, Minas Gerais. Geociências, 34(3): 402-410.

Poff, N.L.; Ward, J.V. 1990. Physical habitat template of lotic systems: Recovery in the context of historical pattern of spatiotemporal heterogeneity. Environmental Management, 14(5): 629-645.

R Development Core Team (2015) R: A language and environment for statistical computing. Vienna, Austria, R Foundation for Statistical Computing.

Rocha, C.H.B.; Pereira, A.M. 2016. Análise multivariada para seleção de parâmetros de monitoramento em manancial de Juiz de Fora, Minas Gerais. Revista Ambiente e Água, 11(1): 176-187.

Santi, G.M.; Furtado, C.M.; Menezes, R.S.A.; Keppeler, E.C. 2012. Variabilidade espacial de parâmetros e indicadores de qualidade da água na sub-bacia hidrográfica do igarapé São Francisco, Rio Branco, Acre, Brasil. Ecología Aplicada, 11(1): 23-31.

Siegloch, A.E; Schmitt, R.; Spies, M.; Petrucio M.; Hernández, M.I.M. Effects of small changes in riparian forest complexity on aquatic insect bioindicators in Brazilian subtropical streams. Marine and Freshwater Research, 68(3): 519-527.

Silva, D.R.O.; Herlihy, A.T.; Hugues, R.M.; Macedo, D.R.; Callisto, M. 2018. Assessing the extent and relative risk of aquatic stressors on stream macroinvertebrate assemblages in the neotropical savanna. Science of the Total Environment. 633: 179-188.

Southwood, T. 1977. Habitat, the templet for ecological strategies? Journal of Animal Ecology, 46: 337-365.

Vannote, R.L.; Minshall, G.W.; Cummins, K.W.; Sedell, J.R.; Cushing, C.E. 1980. The river continuum concept. Canadian Journal of Fisheries and Aquatic Sciences. 37: 130-137. 